\title{
Is Cell-Free Massive MIMO (CF-MMIMO) Equivalent to the Already Existing and Commercialized pCell Technology?
}

\author{
SADIQ IQBAL ${ }^{1}$, JEHAD M. HAMAMREH ${ }^{2}$ \\ ${ }^{1}$ WISLAB, Department of Electrical and Computer Engineering, Antalya Bilim University, Antalya, Turkey (e-mail: sadiq.iqbal@ std.antalya.edu.tr) \\ ${ }^{2}$ WISLAB, Department of Electrical and Electronic Engineering, Antalya Bilim University, Antalya, Turkey (e-mail:jehad.hamamreh@gmail.com) \\ Corresponding author: Sadiq Iqbal (e-mail: sadiq.iqbal@std.antalya.edu.tr)
}

WISLAB (wislabi.com/solutions) offers solutions for building and deploying fully secure, cloud-based, and low-cost end-to-end 4G/5G networks along with providing consultations on helping companies reduce their networks CAPEX/OPEX cost and determine which solutions are best suited for their needs and use cases.

\begin{abstract}
Distributed antenna system (DAS) is a highly diversified concept that can help lower radiated power, maximize coverage, and improve spectral efficiency. Yet, the most exciting feature of DAS is the distribution of remote radio heads (RRH) or antennas over a wide geographical area, which allows the use of less complex signal processing techniques resulting in the reduction of systems' size and cost. These features of DAS were utilized in network MIMO, distributed MIMO, multi-cell MIMO, and distributed-input distributed-output (DIDO) systems to move away from the centralized approach as in conventional multi-user and massive MIMO systems. In this tutorial paper, we first talk about MIMO systems with their relevant technologies by categorizing them into two main approaches according to their working principles and systematic architecture; the first approach is based on a centralized (or collocated) architecture, whereas the second is based on a decentralized (or distributed) architecture. We then deep dive into all the different technologies related to these architectures one by one and explain each in detail with much more focus on the similarities and differences between them. After that, we exposition the main target of this study, which is to answer the following question "Is cell-free massive MIMO (CF-MMIMO) equivalent to the already existing and commercialized pCell technology?". To confidently answer this question, we comprehensively and deeply study the two technologies considering the differences in their system models and look into their conceptual formulations as well as their respective channel models. More particularly, we detail CF-MMIMO and pCell architectures while giving special attention to pCell's SDR wireless platform and stating why it is crucial for the current wireless systems. Then, we present the understandings that are acquired after careful observations and provide recommendations based upon them. In short, this study aims to focus the reader attention on the fact that all the distributed and cellfree MIMO-related technologies work on the same principle, and the way these systems are built makes them fundamentally equivalent, yet, they are displayed quite differently using different terminologies and perspectives, which can cause confusion to readers and end up misleading future researchers.
\end{abstract}

INDEX TERMS MIMO, multi-user MIMO, massive MIMO, network MIMO, distributed MIMO, multicell MIMO, DIDO, cell-free massive MIMO, pCell, 4G, 5G, 6G.

\section{INTRODUCTION}

$\mathbf{I}$ $\mathrm{n}$ wireless communication systems, several interchangeable mutual technologies are available that are pronounced differently but inherently have the same working principle or system architecture. Based on this observation, we focus on the case studies that are innately alike yet are given a different meaning due to how and where they are utilized. In this work, we zoom into the multi-input multi-output (MIMO) system and its counter-parts to see the clear differences they exhibit (based on centralized and de-centralized approaches). As we move forward, we will outline and highlight the details that make these systems comparable to each other but only dissimilar to the meaning of what they portrayed. After that, we move towards the 


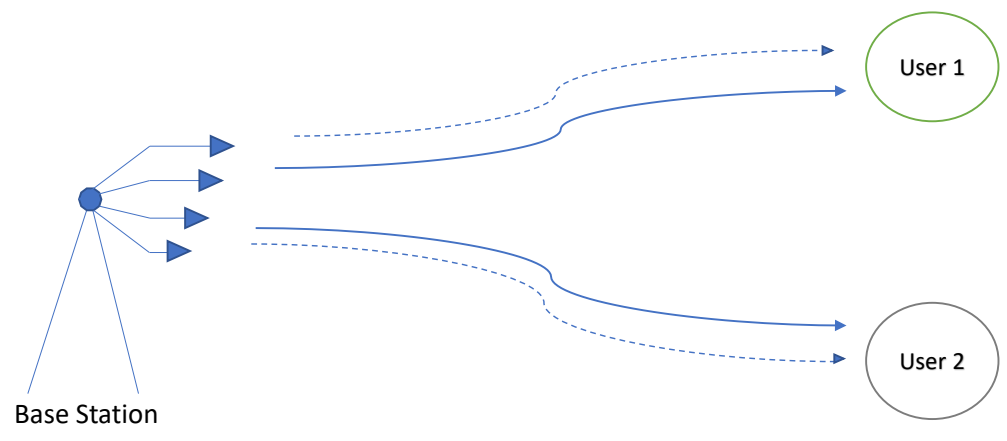

Signal intended for user 1

Signal intended for user 2

FIGURE 1. Multi-user MIMO.

main feature of this work, which covers the section related to CF-MMIMO vs pCell, their comprehensive review, important observations, recommendations, and based on them, we judge "Is CF-MMIMO equivalent to already existing commercialized pCell technology?"

\section{A. RELEVANT MIMO TECHNOLOGIES}

In this section, we briefly discuss the MIMO and its related technologies (classified based on centralized and decentralized methods) that have relevancy among them due to their system architectures and working principles as shown in Tables 1 and 2. Table 1 includes the centralized MIMO approach and Table 2 de-centralized approach.

TABLE 1. Centralized MIMO Technologies

\begin{tabular}{||l||l||}
\hline S.No & \multicolumn{1}{|c||}{ Technology Name } \\
\hline \hline 1 & Multi-user MIMO \\
\hline 2 & Massive MIMO \\
\hline
\end{tabular}

TABLE 2. De-centralized or Distributed MIMO Technologies

\begin{tabular}{||l||l||}
\hline S.No & \multicolumn{1}{|c||}{ Technology Name } \\
\hline \hline 3 & Network MIMO \\
\hline 4 & Distributed MIMO \\
\hline 5 & Multi-cell MIMO \\
\hline 6 & Distributed Input Distributed Output (DIDO) \\
\hline 7 & Joint Transmission CoMP \\
\hline
\end{tabular}

\section{1) Multi-user MIMO}

Multiple-antenna access points can potentially allow higher throughputs, increased diversity, and reduced interference as they communicate with multiple wireless users. It is well known that in a MIMO system with $\mathbf{n}_{\mathbf{T}}$ transmit and $\mathbf{n}_{\mathbf{R}}$ receive antennas capacity grows linearly with $\min \left(\mathbf{n}_{\mathbf{T}}, \mathbf{n}_{\mathbf{R}}\right)$. Similar capacity scaling applies when a $\mathbf{n}_{\mathbf{T}}$ antenna access point communicates with $\mathbf{n}_{\mathbf{R}}$ users [1], [2]. In a multi-user MIMO (MU-MIMO) system, the number of antennas at the base station (BS) is greater than the number of users [1], [3]-[8], and the BS is simultaneously transmitting to the user as shown in Fig. 1.

\section{2) Massive MIMO}

Massive MIMO is an emerging technology that scales up MIMO by possibly orders of magnitude and most particularly, energy efficiency, exploitation of excess degrees of freedom, time-division duplex (TDD) calibration, techniques to combat pilot contamination, and entirely new channel measurements [9]. Massive MIMO relies on spatial multiplexing, which in turn relies on the base station having good channel knowledge, on both the uplink and the downlink scenarios. On the uplink, this is easy to accomplish by having the terminals send pilots, based on which the base station estimates the channel responses to each of the terminals. In the downlink, pilots should be mutually orthogonal between the antennas. Which means that the number of time-frequency resources needed for downlink pilots scales with the number of antennas. In massive MIMO systems, antenna arrays with a few hundred antennas are used that simultaneously serve tens of users in the same temporal-frequency resources [9]-[15]. This makes the number of antennas much larger than the number of users, and the different configurations of the antenna arrays used in massive MIMO are illustrated in Fig. 2.

\section{3) Network MIMO}

The throughput of MIMO networks has been improving at a much slower pace, and the throughput efficiency defined as the ratio of network throughput to PHY bit rate is only around $20 \%$ in 300Mbps 802.11n MIMO networks, and below 10\% in Gbps 802.11ac MU-MIMO networks [16]. As multiplexing gain does not scale to multi-cell WLANs with spatially distributed transmitters. Deploying more access points (APs) does not solve this problem either, as inter-cell contention overhead may undo the intra-cell multiplexing gain from MIMO. 


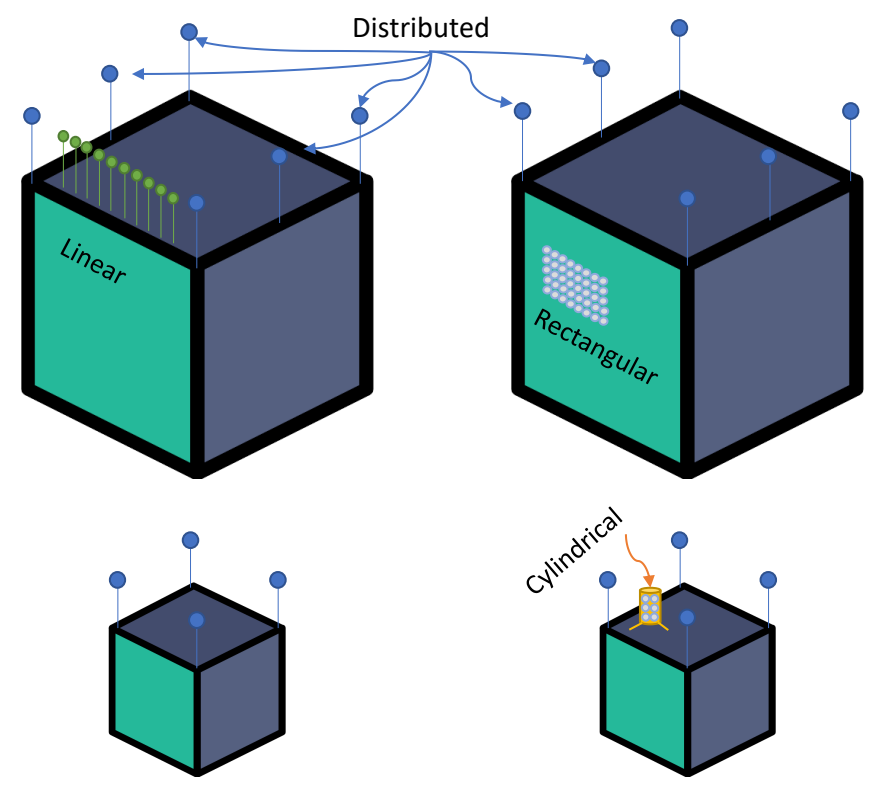

FIGURE 2. Massive MIMO.

Network MIMO (netMIMO) also known as (distributed MU-MIMO) has the potential for eliminating inter-cell interference and contention by allowing APs to synchronize and share the transmission of data packets. An ideal netMIMO the system can enable concurrent transmissions from distributed APs, thus scaling the downlink capacity linearly with the number of APs in a network. APs get synchronized at the carrier signal level by using a reference signal, and share each other's data (for canceling crosstalk interference) via a wireline backhaul [17]. netMIMO can enable concurrent transmissions from distributed APs [17][21]. Fig. 3, illustrates a NEMOx architecture, with a cluster that contains one master AP (mAP) which coordinates a set of distributed APs (dAPs) for netMIMO downlink transmission [18].

\section{4) Distributed MIMO}

In traditional centralized antenna systems, due to the intercell interference, the spectral and power efficiencies are very low at the cell edge [22]. DAS which has been traditionally used to cover the dead spots in indoor wireless communications received attention for its capability of lowering radiated power (and hence other-cell interference), maximizing coverage, and improving spectral efficiency [23]. In DAS, many remote antenna units (RAUs) distributed over a large area and connected to a baseband processing unit (BPU) by fiber, RAUs are only used to transmit and receive signals. At the BPU, the signals associated with different connected RAUs, are processed by using the advanced signal processing techniques, as there is no sophisticated signal processing at RAU, its size and cost are reduced [24].

A generalized DAS was proposed and called a distributed MIMO (D-MIMO) system, these systems combine the advantages of point-to-point MIMO and DAS thus can exploit both spatial micro and macro diversities. Besides collocated MIMO (C-MIMO), D-MIMO system suffers from different degrees of path losses caused by different access distances to different distributed antennas. Thus making the spectral efficiency analysis of D-MIMO more challenging [25], [26]. In Fig. 4, the D-MIMO system is shown, with a generalized cell, where $M$ is the number of antennas of the mobile terminal, $\mathrm{L}$ is the number of antennas per RAU, and $\mathrm{N}$ is the total number of RAUs. In Fig. 4, there are seven RAUs (N =7), and the BPU is located at the central RAU. $\mathrm{NL}$ antennas have a separate feeder to the BPU where all signal processing is performed. When $\mathrm{N}=1$, it becomes a traditional C-MIMO system [24].

\section{5) Multi-cell MIMO}

In a multi-cell scenario, depending on the level of intercell cooperation, the presence of a MIMO broadcast and interference channel can be defined. A simple and analytically traceable model for a multi-cell system with full inter-cell cooperation was introduced by Wyner [27]. In a multi-cell scenario, a cellular system formed by BSs, each equipped with multiple antennas. Each cell contains singleantenna user terminals. Single cell processing and joint twocell processing were investigated by treating the inter-cell interference as Gaussian noise and the flat-fading channel case with full joint cell processing were treated in [28]- 


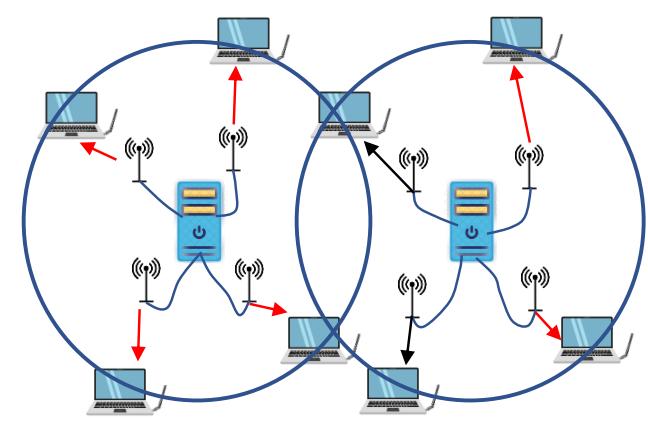

प, Client

mAP (master AP)

(p) dAP (distributed AP)

FIGURE 3. Network MIMO.

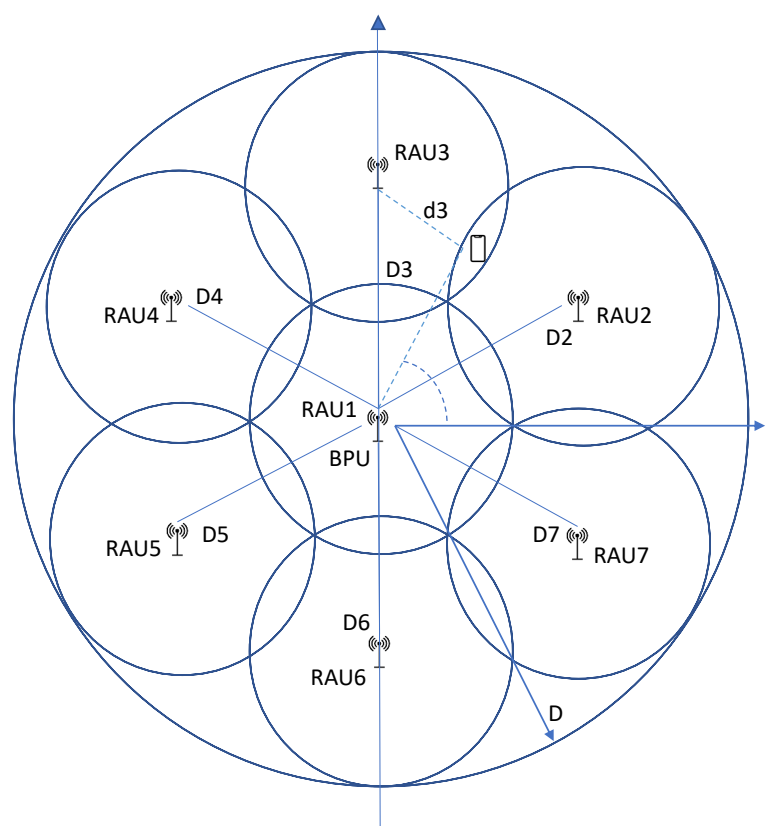

FIGURE 4. Distributed MIMO.

[31]. Fig. 5, illustrates a linear cellular layout with two cells and $\mathbf{K}$ user groups. Suppose $\mathbf{K}=\mathbf{8}$ and assume distance dependent pathloss co-efficients yielding the matrix

$$
\beta=\left[\begin{array}{llllllll}
a & b & b & a & f & e & d & c \\
f & e & d & c & a & b & b & a
\end{array}\right]
$$

for some positive numbers $a, b, c, d, e, f$. The above matrix can be decomposed into four strongly symmetric blocks like

$$
\left[\begin{array}{ll}
a & f \\
f & a
\end{array}\right],\left[\begin{array}{ll}
b & e \\
e & b
\end{array}\right],\left[\begin{array}{ll}
b & d \\
d & b
\end{array}\right],\left[\begin{array}{ll}
a & c \\
c & a
\end{array}\right]
$$

satisfying the above assumption. When these symmetry conditions hold true, the user groups corresponding to the same strongly symmetric block are statistically equivalent, in the sense that they see exactly the same landscape of channel coefficients from all the BSs forming the cluster [28]. 


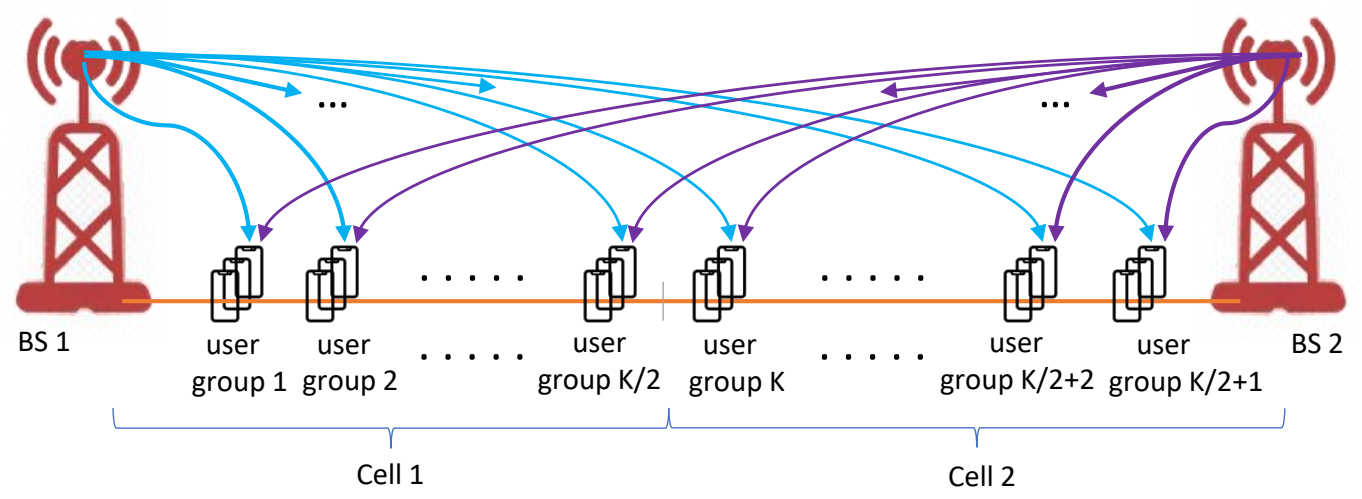

FIGURE 5. Multi-cell MIMO.

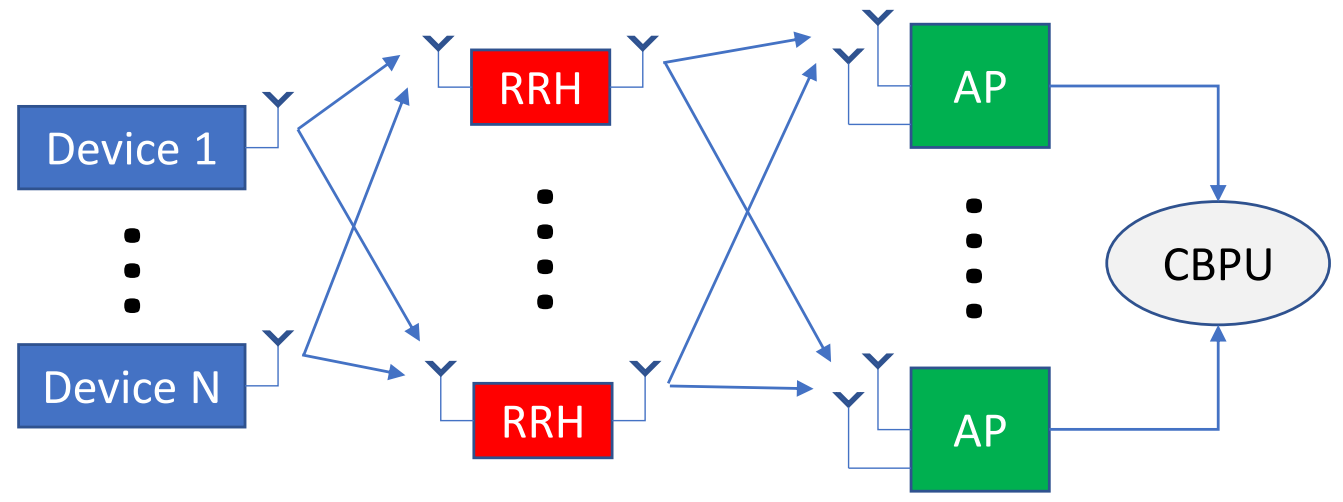

FIGURE 6. Distributed Input Distributed Output Systems.

\section{6) DIDO}

In a cloud radio access network (C-RAN), there are several remote radio heads (RRHs) that are connected to baseband units through fronthaul links. For fronthaul, various options are available, from wireless to wired links (including optical fiber). Among those, wireless front hauls are known to be cheap and flexible, although there might be some challenges, including bandwidth limitations [32]. In C-RAN with twohop. The first hop for the links from devices to RRHs, and the second hop is for links from RRHs to APs. The APs are connected to a central control unit, which is called a central baseband processing unit (CBPU) [32]-[35]. As shown in Fig. 6, the RRHs are connected to multiple APs. In this architecture, the APs are conventional APs that are used for human-type communications (HTC), while the RRHs work as relay nodes for machine-type communications (MTC) so that devices are connected to a network that has limited transmission power by the aid of RRHs and the RRHs are closely located to devices. The resulting system model can be stated as a distributed input distributed output (DIDO) system consisting of several RRHs and multiple APs [32].

\section{7) Joint Transmission CoMP}

Joint Transmission (JT) CoMP consists of different coordinating nodes of the CoMP cluster that can coherently or noncoherently transmit the same data to a the particular user equipment (UE), also by using phase information between the transmission nodes, it becomes possible for the network to mitigate inter-user interference within the CoMP cluster [36]-[39]. In the C-RAN realization of JT CoMP, the CPU is able to control the RRH nodes of the same CoMP cluster as shown in Fig. 7, where inter-user interference between three users is canceled via joint beamforming across multiple nodes performed at the CPU and the channel state and data information for all users is exchanged between the coordinated nodes and the CPU by using high capacity, low latency backhaul links [36].

According to the earlier discussions, the system models presented relate to MIMO technologies that are based on centralized (collocated or undistributed) and de-centralized (distributed) architectures, it is apparent that the decentralized technologies are comparable to each other thus mostly only differ in name. 


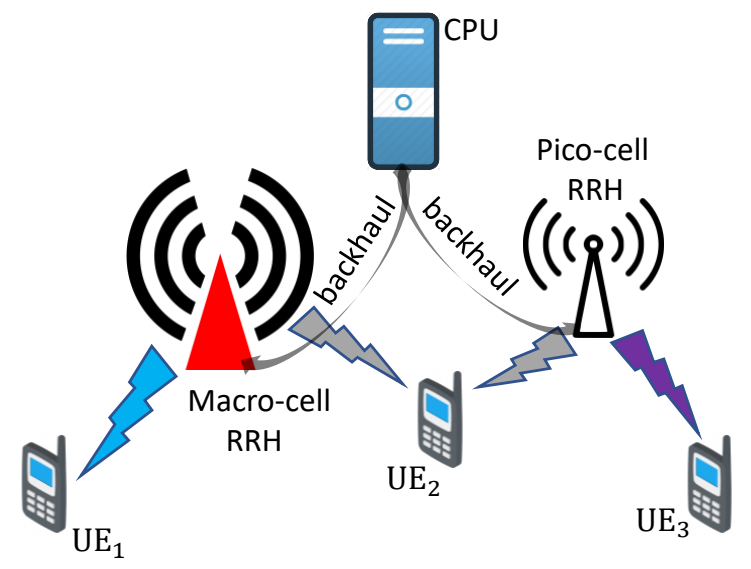

FIGURE 7. Joint Transmission CoMP.

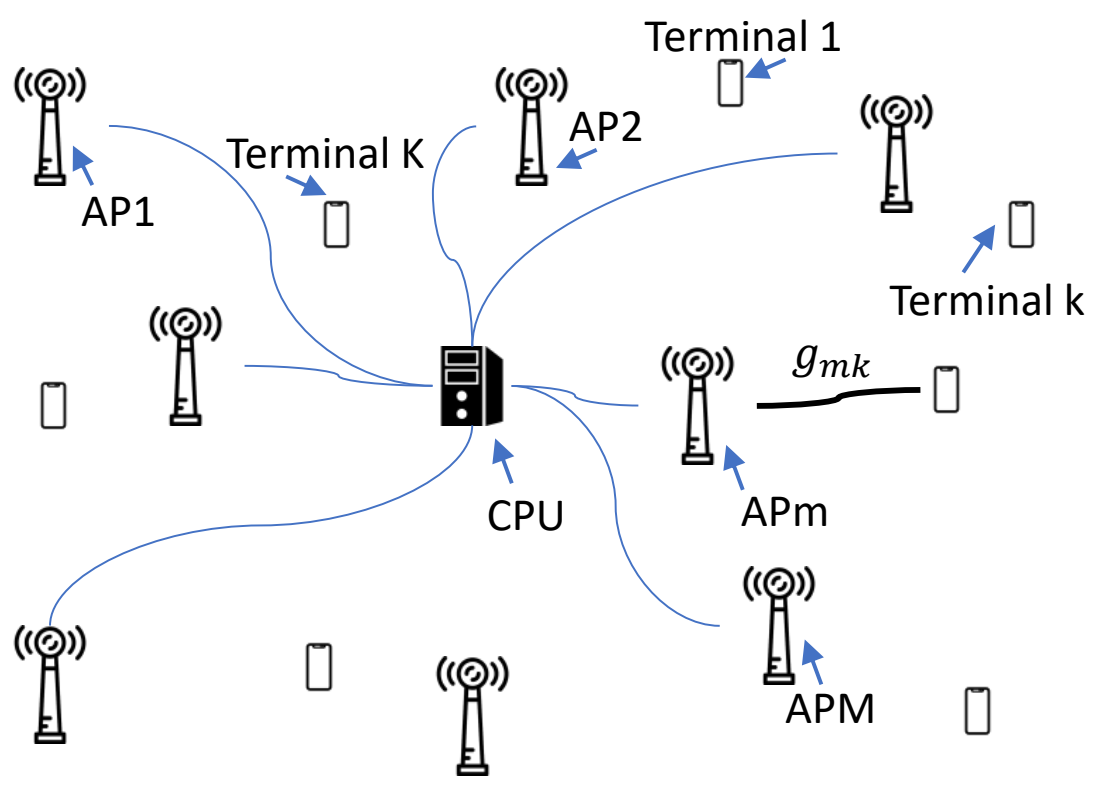

FIGURE 8. CF-MMIMO.

\section{B. IMPORTANT DIFFERENCES}

From Table 1, we can point out the difference between multi-user and massive MIMO, the antennas are located at the BS, which makes them centrally located and essentially termed as collocated or centralized or C-MIMO [1], [9]. On the other hand, Table 2 summarizes the technologies that utilize the DAS approach, and no antennas are centrally located at the BS instead are distributed, which makes them de-centralized or distributed or D-MIMO [18], [24], [28], [32].

\section{The comparable key points:}

1) The number of BSs (APs) and antennas are greater than number of users.

2) Most of the before-mentioned technologies use DAS analogy with C-RAN architecture that has RRH, BBU, CPU and a back-haul link.
3) All the transmissions are done concurrently to all the users.

\section{CF-MMIMO VS PCELL TECHNOLOGY}

Moving towards the main objective of this paper the CFMMIMO $v s$ pCell. What are CF-MMIMO and pCell technologies? Given in the below sections are the comprehensive details regarding them and subsequently, we compare the two technologies according to obtained observations and challenge the relevant authors through the current understanding of the similarities and dissimilarities between the two system models and denounce the claims provided in the relevant papers. Even more so, we came across a very delusional and unconvincing approach of not citing the highly relevant technologies in both of the papers concerning CFMMIMO [40]-[44] and pCell [45]-[48], this leads us to 


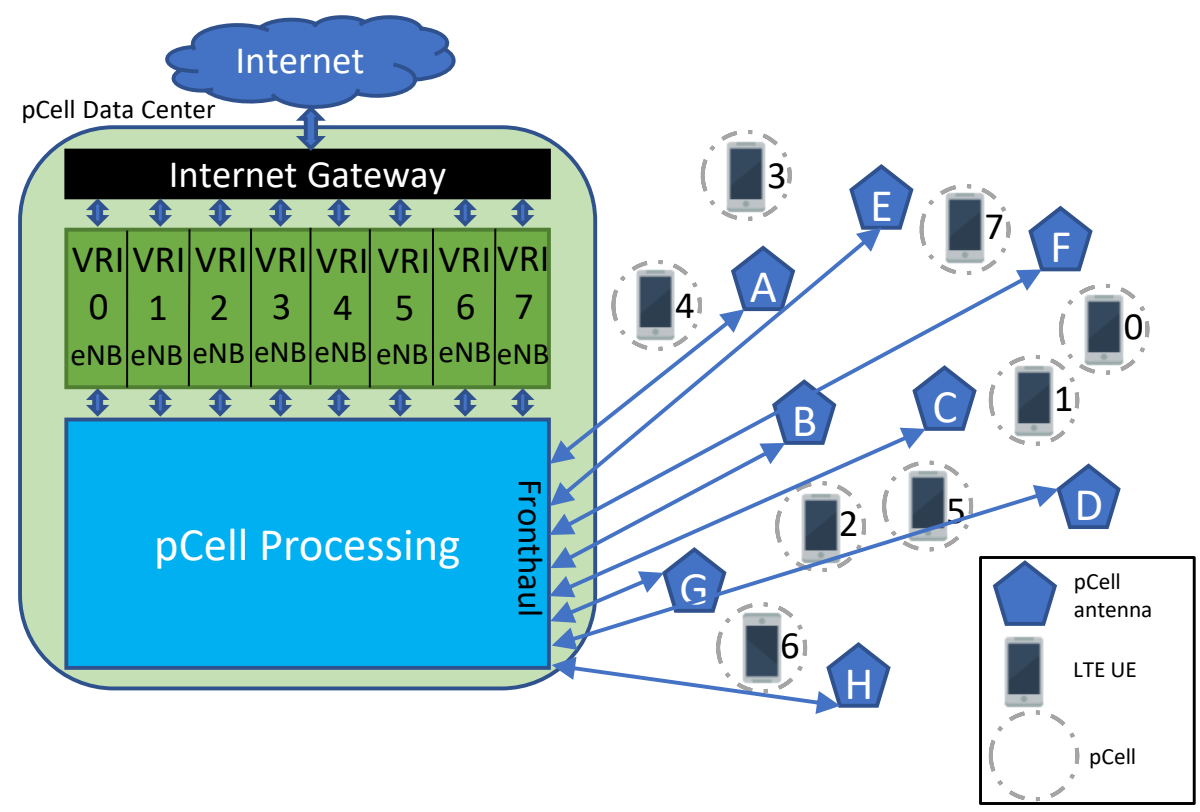

FIGURE 9. pCell software architecture.

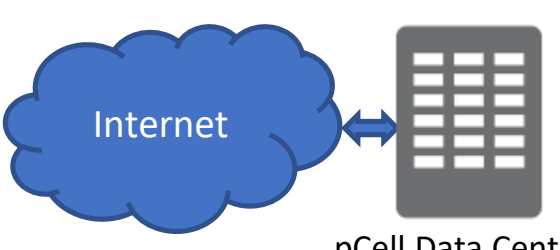

pCell Data Center

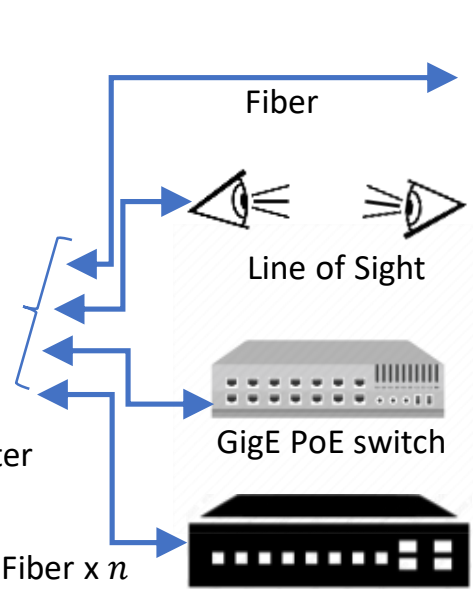

Artmeis Hub
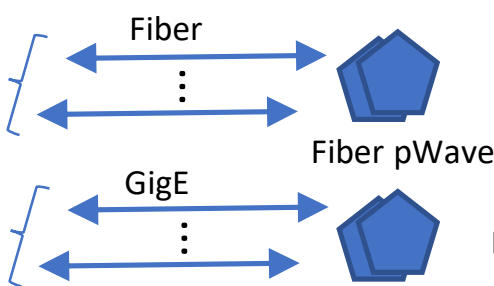

LTE UES
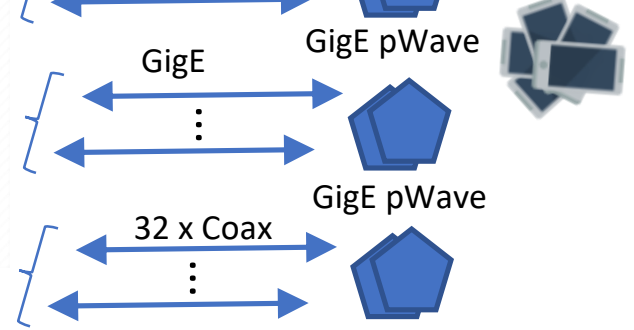

Antennas

FIGURE 10. pCell hardware architecture.

think that the corresponding authors are not enlightened towards the other extraordinary works in the literature that are on par with their technology.

\section{1) What is CF-MMIMO?}

Massive antenna arrays, when arranged in a collocated or distributed fashion at the BS, result in a different structural arrangement, such as a collocated scheme, where the service antennas situated in a compact area result in low-backhaul requirements, moreover, in the distributed model, the service antennas spread over a large area which increases the back- haul requirements. Yet, this strategy increases the diversity exploitation against shadow fading, resulting in the surge of coverage area than collocated structural arrangement [44]. A CF-MMIMO does not have cells or cell boundaries and where a large number of distributed antennas called APs, serve a much smaller number of independent users dispersed over a wide area. A backhaul network is used for the APs to phase-coherently cooperate and serve the users over the same time-frequency resources via time-division duplex (TDD) operation [40], [44].

As APs are scattered, this makes the users closer to them, 


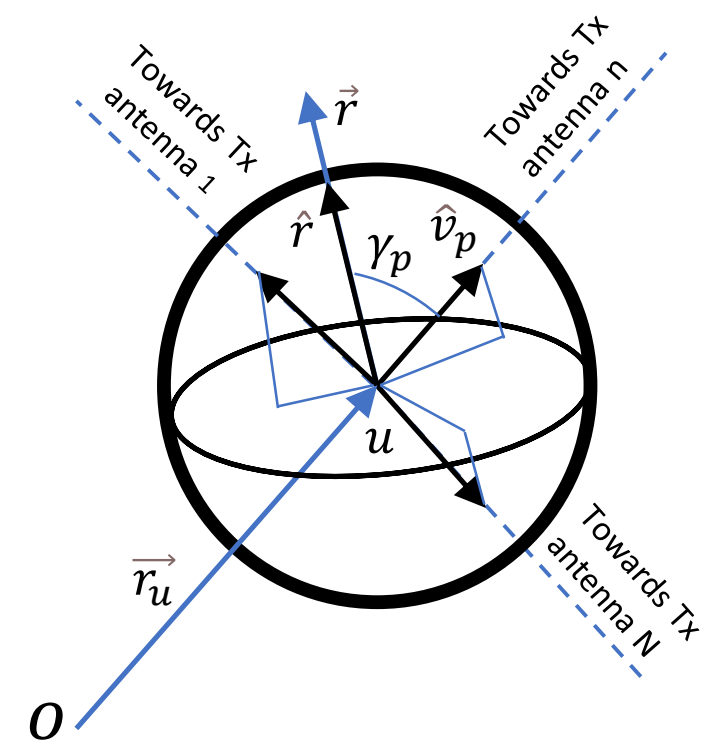

FIGURE 11. Model parameters for LOS channels.

now CF-MMIMO can provide higher coverage and utilize signal processing techniques like conjugate beamforming (known as maximum ratio processing) in a distributed fashion with the whole processing done locally at the APs. CFMMIMO also includes a CPU, yet the information exchange between the APs and this CPU is limited to the payload data and the power control coefficients that slowly change. Also, there is no sharing of instantaneous channel state information (CSI) among the APs or the CPU. All estimated values of channels are present at the APs, obtained through uplink pilots and the channel estimates are used to precode the transmitted data in the downlink and to perform data detection in the uplink [44].

The working principle of CF-MMIMO is an excellent embodiment of design concepts like netMIMO, D-MIMO, CoMP, and DAS. To take advantage of phenomenons like favorable propagation and channel hardening CF-MMIMO employs a substantial amount of single antenna APs, that serve a minuscule amount of users by doing simple signal processing. In Fig. 8, CF-MMIMO system is illustrated, that includes randomly located M APs and K users each equipped with a single antenna, the APs connect to a CPU via a backhaul network and serve the $\mathrm{K}$ users simultaneously over the same time/frequency. The uplink and downlink transmissions occur in TDD operation, and the channel estimates obtained during the uplink pilot training phase are used to precode the signals during downlink transmission. For users, it is not mandatory to estimate the channel gains, instead hinge on channel hardening to improve the gain value [44].

CF-MMIMO also incorporates the following assumptions like: 1) the effects of small and large scale fading taken into consideration in the design of channel model, the small scale fading is static in each coherence interval and changes from one interval to another, on the contrary, large scale fading is slow and stays constant for several coherence intervals [44]; 2) TDD operation is utilized to get a reciprocal channel gain, so this means the channel gains in the uplink and downlink are the same; 3) When modeling a channel denoted by $g_{m k}$, representing the channel coefficient between $m$ th AP and the $k$ th user, it is given as:

$$
g_{m k}=\beta_{m k}^{1 / 2} h_{m k},
$$

where $h_{m k}$ represents the small scale fading, $\beta_{m k}^{1 / 2}$ as the large scale fading and in $h_{m k}, m=1, \ldots, M, K=1, \ldots, K$ don't depend on each other and are identical.

\section{2) What is pCell?}

A MU-MIMO in its simplest form consists of $N$ transmit antennas and $U$ single-antenna receivers. However, considering real-world systems over theoretical models gives rise to many practical limitations thus, centralized antenna structures yield low spatial diversity because the performance of MIMO systems relies on the limited multi-paths available in the propagation channel. The solution to the problem is to deploy a much larger number of antennas than users to get enhanced spatial diversity (as in massive MIMO) and provide a more independent spatial channel to multiconcurrent users by beamforming techniques. However, this arrangement limits the degree of freedom because of highly complex BS with tightly packed RF chains with a centralized antenna system [45]. In contrast, the de-centralized cellular architecture can achieve the same spectral efficiency as in massive MIMO with the one order of magnitude fewer antennas by utilizing netMIMO and cooperating with BS of 
adjacent cells grants inter-cell interference-free communication to improve cell edge performance.

To radically improve SE commercially deployable pCell technology was introduced and can achieve SE over an order of magnitude higher than the 4G LTE technology. pCell obtains these gains by discarding cellularization and benefiting from interference in wireless networks. The benefit acquired by exploiting interference through large-scale cooperation between distributed transceivers and also by allowing high multiplexing gain via multi-user transmissions [45]. As transmit power increases, SE reaches an upper limit because of out-of-cluster interference affecting in-cluster signals, and this counts as a fundamental capacity limitation in cooperating networks. In pCell, a different architecture implementation is carried out, with transceivers distributed independently, there is no such thing as cell consideration in pCell, and to increase spatial multiplexing gain high densification is exploited with fixed transmit power. Fronthaul is used as a medium for large-scale cooperation that creates concurrent spatial channels to multiple users through precoding [45].

The channel model concerning pCell is responsible for spatial dependency of the electromagnetic field between transmit and receive antennas. By utilizing this channel, the notion of volumes in space of coherent signal is designed, and point antennas are employed that are un-polarized and isotropic radiators in far fields. The environment considered is in terms of scatterers and models of spherical waves. Also, another noted assumption is that the distance between users is much smaller than their distance to transmitters and scatterers [45]. Therefore, the model of the complex channel coefficient between transmit antenna $n=1, \ldots, N$ and receive antenna $u=1, \ldots, U$ is the location vector of a superposition of plane waves and is given as:

$$
h_{u n}=\sum_{p \in S_{u n}} a_{p} e^{-i k \hat{v}_{p} \cdot \vec{r}_{u}}
$$

where $S_{u n}$ is the set of all paths, including line of sight, non-line-of-sight, propagation and cluster scattering from transmit antenna $n$ to receive antenna $u$, while $k=2 \pi / \lambda$ is the wave number, $\lambda$ is the wavelength. As shown in Fig. 11, $\vec{r}_{u}$ is the location vector of user $u$ relative to an origin $O$, $\hat{v}_{p}$ is the unit vector in the direction of the incident path $p$ pointing out from the location of user $u, a_{p}$ is the complex coefficient designing pathloss, shadowing and phase terms independent of $\vec{r}_{u}$ for path $p$.

pCell SDR Wireless Platform: The pCell softwaredefined radio (SDR) wireless platform performs baseband processing in a data center which makes it an imitation of CRAN, and this SDR platform consists of two parts hardware and software as described below:

1) Hardware Architecture:

In the hardware section, as illustrated in Fig. 10, the pCell system includes two parts: i) a general-purpose processor (GPP) based data center that executes LTE protocol and pCell processing; and ii) a RAN that contains data switches and radio transceivers. As shown in Fig. 10, a fiber fronthaul transmits I/Q duplex digital sample streams, which are routed by servers in the data center and can be connected with:

- pWaves that are configured with a fiber interface.

- LOS radios that connect to pWaves configured for 1000BASE-T (copper Gigabit Ethernet).

- an uplink port of 1000BASE-T swith that is configured for 1000BASE-T.

- An Artemis Hub integrated with 32 pWave radios that connect to 32 antennas via coaxial cables.

2) Software Architecture: The software architecture helps reduce computational overhead, optimize throughput and provide computational load behavior, which is deterministic and stable, and to obtain maximum flexibility, interoperability, and portability, the pCell SDR implementation is one of the best options. Why is that? Because this platform has all the functional blocks of the LTE protocol stack (from the gateway down to the physical layer) with the pCell processing module that has been built from scratch in $\mathrm{C}++$. There is no need for special hardware like DSPS, co-processors, or FPGAs [45].

The software modules are classified into branches like hard real-time and soft real-time to achieve the expected efficiency for the real-time constraints due to LTE protocol and pCell processing. To complete the operations like pCell processing, LTE physical layer functions such as turbo coding and decoding, FFT, channel estimation/equalization, and some MAC layer functionality like PRACH procedure on time is of critical importance for maintaining the integrity of the pCell-synchronized LTE waveform and the stability of data connection with the UE. The period of completion of these tasks should be the fraction of $1 \mathrm{~ms}$ LTE sub-frame (SF) duration. The higher layers of the LTE protocol stack (like RLC, PDCP, RRC, NAS, Gateways) can be implemented as functional blocks in the soft real-time modules, and these blocks are under the effect of time constraints that are an order of multiples of an SF interval. By following the above classification and the tools provided by the development framework, the system can guarantee on-time execution of the critical hard real-time tasks without leaving the deadlines of the soft real-time ones unattended.

In Fig. 9 the software architecture of pCell is shown, for every UE, a virtual radio instance (VRI) is allocated (containing a set of computing resources and data structure) that acts as an entire LTE protocol 
stack, allowing the UE to experience the functionalities which an LTE eNodeB provides. The procedure of VRI initialization follows the UE attachment and detachment. It remains in the active state until the UE is connected to the network. The VRI is released after the VRI manager saves the relevant state as the user detaches from the system. The VRI is linked to the volume of coherent signals around the user through the physical layer signal processing. Thus, every VRI has a whole bandwidth with an independent physical layer link, allowing the user to encounter the experience of an unshared eNodeB.

As illustrated in Fig. 9, the spatial processing function indicated as pCell processing and the volumes of the coherent signal represented by the dashed circles around the UE with the numeric labels matching the respective associated VRI link. Talking about VRIs, they have their functionalities like some VRIs can implement LTE eNodeB protocol and others can implement standard protocols. Both LTE and non-LTE devices can simultaneously and individually operate in the same spectrum and each device experiences the protocol from its own VRI.

The above detailed description about pCell increases its importance and actually shows why pCell technology is game changing in the relevant field of wireless communication systems.

\section{3) Comparison between CF-MMIMO and pCell}

1) CF-MMIMO and pCell both use a distributed antenna approach.

2) There are no cells present in CF-MMIMO and pCell.

3) Both CF-MMIMO and pCell employ a CPU approach that is connected with APs.

4) CF-MMIMO and pCell both utilizes backhaul approach.

5) CF-MMIMO uses channel hardening but has three training phases while pCell also utilizes channel hardening concept.

6) CF-MMIMO uses conjugate beamforming/matched filtering techniques but pCell uses transmit precoding techniques such as linear zero forcing. This clearly shows that both the technologies utilize precoding as one of the main ingredients.

7) CF-MMIMO utilizes small scale fading and large scale fading in its channel model [44], while pCell employs a geometrical spatial channel model to define volumes in space of coherent signal around the user antenna [45].

Observations: The above-mentioned main comparison highlights the differences and similarities between CFMMIMO and pCell. But we also noticed that CF-MMIMO has no critical differences than pCell that makes it stand out. Because pCell firstly utilizes the design of a geometrical model, including volumes in space of coherent signals considering the model of spherical waves in a scattering environment, which results in the volumes in space of coherent signals around the users that are also called pcells [45] and secondly, is the commercialized implementation of pCell's own hardware and software architecture in the LTE protocol.

Next in line, we will point out the habit of not citing the relevant paper-like [45] in the corresponding paper such as [44] or vice versa, because these papers are incredibly similar to each other, so they should be included in the relevant works. To finish this discussion, we bring to the table a verity, that the technologies such as netMIMO, D-MIMO, multi-cell MIMO, DIDO, JT-CoMP, CF-MMIMO, and pCell all utilize the concept of DAS (that has a CPU, distributed APs with randomly located number of users), which makes them comparable to each other. Nevertheless, these technologies are pronounced differently from each other and the authors do not mention the other relevant studies. This acts as a misguidance towards the readers.

Recommendations: By providing the previous distinctive facts, the main goal of this study is to inform the readers about the concept used in [44], [45]. CF-MMIMO and pCell, in reality, are identical, so this leads us to push the claim like "CF-MMIMO authors should cite pCell and treat it as a benchmark for future works." The practicality of the incredible work should be highly appreciated that the pCell team has done [45].

\section{CONCLUSION}

In this work, we have presented a framework that classifies the MIMO systems, their related technologies according to their system architecture approaches and proves that CFMMIMO is equivalent to already existing pCell technology. The primary purpose of this study is to clear the concept of DAS and distributed MIMO technologies so that the readers can easily understand the differences between them and the ideology on which the statement "Are CF-MMIMO and pCell related to each other or not?" is based. Explicitly, the framework has two sections: the first section is dedicated to MIMO-related technologies, was further divided into two portions that are associated with centralized and decentralized MIMO technologies accompanied with their description and system models, respectively. The second section includes the differences between the relevant MIMO technologies and their comparable points. After this, we focus on the foremost part of the paper, which encompasses the discussions related to CF-MMIMO and the pCell. First, we defined them comprehensively with their respective system models, then compared them with each other, the comparison includes the principal features of CF-MMIMO and pCell that make them equivalent to each other. The paper has been culminated by providing the observations and recommendations relevant to CF-MMIMO and pCell. 


\section{REFERENCES}

[1] Q. H. Spencer, C. B. Peel, A. L. Swindlehurst, and M. Haardt, "An introduction to the multi-user MIMO downlink," IEEE communications Magazine, vol. 42, no. 10, pp. 60-67, 2004.

[2] H. Weingarten, Y. Steinberg, and S. Shamai, "The capacity region of the gaussian MIMO broadcast channel," in International Symposium onInformation Theory, 2004. ISIT 2004. Proceedings. IEEE, 2004, p. 174.

[3] A. Goldsmith, S. A. Jafar, N. Jindal, and S. Vishwanath, "Capacity limits of MIMO channels," IEEE Journal on selected areas in Communications, vol. 21, no. 5, pp. 684-702, 2003.

[4] E. Björnson, L. Sanguinetti, J. Hoydis, and M. Debbah, "Optimal design of energy-efficient multi-user MIMO systems: Is massive MIMO the answer?" IEEE Transactions on wireless communications, vol. 14, no. 6, pp. 3059-3075, 2015.

[5] V. Stankovic and M. Haardt, "Generalized design of multi-user MIMO precoding matrices," IEEE Transactions on Wireless Communications, vol. 7, no. 3, pp. 953-961, 2008.

[6] E. Björnson, L. Sanguinetti, J. Hoydis, and M. Debbah, "Designing multi-user MIMO for energy efficiency: When is massive MIMO the answer?" in 2014 IEEE wireless communications and networking conference (WCNC). IEEE, 2014, pp. 242-247.

[7] B. Gopalakrishnan and N. Jindal, "An analysis of pilot contamination on multi-user MIMO cellular systems with many antennas," in 2011 IEEE 12th international workshop on signal processing advances in wireless communications. IEEE, 2011, pp. 381-385.

[8] O. Bejarano, E. W. Knightly, and M. Park, "Ieee 802.11 ac: from channelization to multi-user MIMO," IEEE Communications Magazine, vol. 51, no. 10, pp. 84-90, 2013.

[9] E. G. Larsson, O. Edfors, F. Tufvesson, and T. L. Marzetta, "Massive MIMO for next generation wireless systems," IEEE communications magazine, vol. 52, no. 2, pp. 186-195, 2014.

[10] F. Rusek, D. Persson, B. K. Lau, E. G. Larsson, T. L. Marzetta, O. Edfors, and F. Tufvesson, "Scaling up MIMO: Opportunities and challenges with very large arrays," IEEE signal processing magazine, vol. 30, no. 1, pp. 40-60, 2012.

[11] J. Nam, J.-Y. Ahn, A. Adhikary, and G. Caire, "Joint spatial division and multiplexing: Realizing massive MIMO gains with limited channel state information," in 2012 46th annual conference on information sciences and systems (CISS). IEEE, 2012, pp. 1-6.

[12] C. Shepard, H. Yu, N. Anand, E. Li, T. Marzetta, R. Yang, and L. Zhong, "Argos: Practical many-antenna base stations," in Proceedings of the 18th annual international conference on Mobile computing and networking, 2012, pp. 53-64.

[13] X. Gao, F. Tufvesson, O. Edfors, and F. Rusek, "Measured propagation characteristics for very-large MIMO at $2.6 \mathrm{ghz}$," in 2012 Conference Record of the Forty Sixth Asilomar Conference on Signals, Systems and Computers (ASILOMAR). IEEE, 2012, pp. 295-299.

[14] J. Hoydis, S. Ten Brink, and M. Debbah, "Massive MIMO in the UL/DL of cellular networks: How many antennas do we need?" IEEE Journal on selected Areas in Communications, vol. 31, no. 2, pp. 160-171, 2013.

[15] A. Lozano, R. W. Heath, and J. G. Andrews, "Fundamental limits of cooperation," IEEE transactions on information theory, vol. 59, no. 9, pp 5213-5226, 2013.

[16] K. Tan, J. Fang, Y. Zhang, S. Chen, L. Shi, J. Zhang, and Y. Zhang, "Finegrained channel access in wireless LAN," in Proceedings of the ACM SIGCOMM 2010 conference, 2010, pp. 147-158.

[17] D. Gesbert, S. Hanly, H. Huang, S. S. Shitz, O. Simeone, and W. Yu, "Multi-cell MIMO cooperative networks: A new look at interference," IEEE journal on selected areas in communications, vol. 28, no. 9, pp 1380-1408, 2010.

[18] X. Zhang, K. Sundaresan, M. A. Khojastepour, S. Rangarajan, and K. G Shin, "NEMOx: Scalable network MIMO for wireless networks," in Proceedings of the 19th annual international conference on Mobile computing \& networking, 2013, pp. 453-464.

[19] H. V. Balan, R. Rogalin, A. Michaloliakos, K. Psounis, and G. Caire "Achieving high data rates in a distributed MIMO system," in Proceedings of the 18th annual international conference on Mobile computing and networking, 2012, pp. 41-52.

[20] S. Gollakota, S. D. Perli, and D. Katabi, "Interference alignment and cancellation," in Proceedings of the ACM SIGCOMM 2009 conference on Data communication, 2009, pp. 159-170.

[21] K. C.-J. Lin, S. Gollakota, and D. Katabi, "Random access heterogeneous MIMO networks," ACM SIGCOMM Computer Communication Review, vol. 41, no. 4, pp. 146-157, 2011.
[22] X. You, D. Wang, P. Zhu, and B. Sheng, "Cell edge performance of cellular mobile systems," IEEE Journal on Selected Areas in Communications, vol. 29, no. 6, pp. 1139-1150, 2011.

[23] A. A. Saleh, A. Rustako, and R. Roman, "Distributed antennas for indoor radio communications," IEEE Transactions on Communications, vol. 35 no. 12, pp. 1245-1251, 1987.

[24] D. Wang, J. Wang, X. You, Y. Wang, M. Chen, and X. Hou, "Spectra efficiency of distributed MIMO systems," IEEE Journal on Selected Areas in Communications, vol. 31, no. 10, pp. 2112-2127, 2013.

[25] W. Roh and A. Paulraj, "MIMO channel capacity for the distributed antenna," in Proceedings IEEE 56th Vehicular Technology Conference, vol. 2. IEEE, 2002, pp. 706-709.

[26] W. Choi and J. G. Andrews, "Downlink performance and capacity of distributed antenna systems in a multi-cell environment," IEEE transactions on wireless communications, vol. 6, no. 1, pp. 69-73, 2007.

[27] A. D. Wyner, "Shannon-theoretic approach to a Gaussian cellular multipleaccess channel," IEEE Transactions on Information Theory, vol. 40, no. 6 , pp. 1713-1727, 1994

[28] H. Huh, S.-H. Moon, Y.-T. Kim, I. Lee, and G. Caire, "Multi-cell MIMO downlink with cell cooperation and fair scheduling: A large-system limit analysis," IEEE Transactions on Information Theory, vol. 57, no. 12, pp. 7771-7786, 2011.

[29] O. Somekh, B. M. Zaidel, and S. Shamai, "Sum rate characterization of joint multiple cell-site processing," IEEE Transactions on Information Theory, vol. 53, no. 12, pp. 4473-4497, 2007.

[30] S. Shamai and A. D. Wyner, "Information-theoretic considerations for symmetric, cellular, multiple-access fading channels. i," IEEE Transactions on Information Theory, vol. 43, no. 6, pp. 1877-1894, 1997.

[31] R. Irmer, H. Droste, P. Marsch, M. Grieger, G. Fettweis, S. Brueck, H.-P Mayer, L. Thiele, and V. Jungnickel, "Coordinated multipoint: Concepts, performance, and field trial results," IEEE Communications Magazine, vol. 49, no. 2, pp. 102-111, 2011.

[32] J. Choi, "Compressive random access for MTC in distributed input distributed output systems," in 2017 IEEE 85th Vehicular Technology Conference (VTC Spring). IEEE, 2017, pp. 1-5.

[33] S.-H. Park, O. Simeone, O. Sahin, and S. S. Shitz, "Fronthaul compression for cloud radio access networks: Signal processing advances inspired by network information theory," IEEE Signal Processing Magazine, vol. 31 , no. 6, pp. 69-79, 2014.

[34] G. Wunder, P. Jung, and C. Wang, "Compressive random access for postLTE systems," in 2014 IEEE International Conference on Communications Workshops (ICC). IEEE, 2014, pp. 539-544.

[35] J. N. Laneman, D. N. Tse, and G. W. Wornell, "Cooperative diversity in wireless networks: Efficient protocols and outage behavior," IEEE Transactions on Information theory, vol. 50, no. 12, pp. 3062-3080, 2004

[36] A. Davydov, G. Morozov, I. Bolotin, and A. Papathanassiou, "Evaluation of joint transmission CoMP in C-RAN based LTE-A HetNets with large coordination areas," in 2013 IEEE Globecom Workshops (GC Wkshps). IEEE, 2013, pp. 801-806.

[37] A. Barbieri, P. Gaal, S. Geirhofer, T. Ji, D. Malladi, Y. Wei, and F. Xue "Coordinated downlink multi-point communications in heterogeneous cellular networks," in 2012 Information Theory and Applications Workshop. IEEE, 2012, pp. 7-16.

[38] G. Li, S. Zhang, X. Yang, F. Liao, T.-f. Ngai, S. Zhang, and K. Chen, "Architecture of GPP based, scalable, large-scale C-RAN BBU pool," in 2012 IEEE Globecom Workshops. IEEE, 2012, pp. 267-272.

[39] B. Clerckx, G. Kim, J. Choi, and Y.-J. Hong, "Explicit vs. implicit feedback for SU and MU-MIMO," in 2010 IEEE Global Telecommunications Conference GLOBECOM 2010. IEEE, 2010, pp. 1-5.

[40] E. Nayebi, A. Ashikhmin, T. L. Marzetta, and H. Yang, "Cell-free massive MIMO systems," in 2015 49th Asilomar Conference on Signals, Systems and Computers. IEEE, 2015, pp. 695-699.

[41] E. Nayebi, A. Ashikhmin, T. L. Marzetta, H. Yang, and B. D. Rao, "Precoding and power optimization in cell-free massive MIMO systems,' IEEE Transactions on Wireless Communications, vol. 16, no. 7, pp. 44454459, 2017.

[42] E. Nayebi, A. Ashikhmin, T. L. Marzetta, and B. D. Rao, "Performance of cell-free massive MIMO systems with MMSE and LSFD receivers,' in 2016 50th Asilomar Conference on Signals, Systems and Computers. IEEE, 2016, pp. 203-207.

[43] H. Q. Ngo, A. Ashikhmin, H. Yang, E. G. Larsson, and T. L. Marzetta, "Cell-free massive MIMO: Uniformly great service for everyone," in 2015 IEEE 16th international workshop on signal processing advances in wireless communications (SPAWC). IEEE, 2015, pp. 201-205. 
[44] Q. Ngo, Hien, A. Ashikhmin, H. Yang, Larsson, G. Erik, and T. L. Marzetta, "Cell-free massive MIMO versus small cells," IEEE Transactions on Wireless Communications, vol. 16, no. 3, pp. 1834-1850, 2017.

[45] A. Forenza, S. Perlman, F. Saibi, M. Di Dio, R. Van Der Laan, and G. Caire, "Achieving large multiplexing gain in distributed antenna systems via cooperation with pCell technology," in 2015 49th Asilomar Conference on Signals, Systems and Computers. IEEE, 2015, pp. 286293.

[46] A. Bleicher, "5G service on your 4G phone?[news]," IEEE Spectrum, vol. 51, no. 4, pp. 13-18, 2014.

[47] I. Akbar, "Power from the void?: How steve perlman's" revolutionary" wireless technology works and why it is a bigger deal than anyone realizes." IEEE Consumer Electronics Magazine, vol. 3, no. 3, pp. 36-43, 2014.

[48] J. Harris, "An artful solutiion," Electrical Connection, no. Summer 2016, pp. $38-40,2016$.

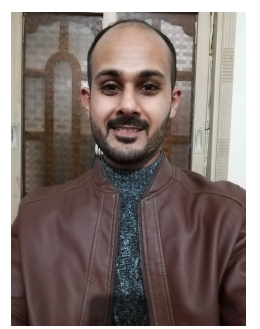

SADIQ IQBAL received the B.E. degree in electrical engineering from QUEST University Nawabshah, Pakistan in 2016. He is presently pursuing the master's (M.Sc.) degree in electrical and computer engineering. He is currently with Antalya Bilim University, Turkey.

His research interests include physical layer technology, 5G communication networks, artificial intelligence, IoT and its applications..

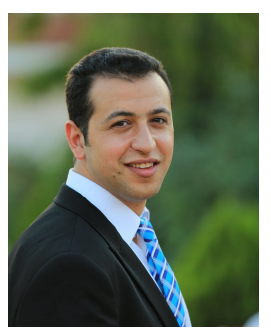

JEHAD M. HAMAMREH is the Founder and Director of WISLAB (wislabi.com), and A. Professor with the Electrical and Electronics Engineering Department, Antalya Bilim University. He received his Ph.D. degree in telecommunication engineering and cyber systems from Istanbul Medipol University, Turkey, in 2018. Previously, he worked as a Researcher at the Department of Electrical and Computer Engineering at Texas AM University. He is the inventor of more than $20+$ Patents and an author of more than $85+$ peer-reviewed scientific papers along with several book chapters. His innovative patented works won the gold, silver, and bronze medals by numerous international invention contests and fairs.

His current research interests include wireless physical and MAC layers security, orthogonal frequency-division multiplexing and multiple-input multiple-output systems, advanced waveforms design, multidimensional modulation techniques, and orthogonal/non-orthogonal multiple access schemes for future wireless systems. He is a serial referee for various scientific journals as well as a TPC member for several international conferences. He is an Editor at Researcherstore, RS-OJICT journal, and Frontiers in Communications and Networks. Email: jehad.hamamreh@gmail.com. 